\title{
Constitutive Modeling of Phase Transformation and Plastic Yield in SMAs: Application to the S3T-RoundRobin
}

\author{
D. Hartl ${ }^{1}$, S. Oehler ${ }^{1}$, and D.C. Lagoudas ${ }^{1, a}$ \\ Department of Aerospace Engineering, Texas A\&M University, College Station, TX, USA
}

\begin{abstract}
In collaboration with other researchers contributing to the S3T-RoundRobin effort, this work presents analytical results as generated using the model of Hartl and Lagoudas (Hartl and Lagoudas, 2009; Hartl et al., 2009). The model, which is based on continuum thermodynamics and inspired by experimental findings, is briefly summarized. Its calibration to the current experimental results is described and predictions generated by the implemented model are compared to the shared data. Some inaccuracies are observed in the uniaxial responses, though good agreement is demonstrated overall, especially with regard to the non-proportional tension-torsion experiments.
\end{abstract}

\section{Constitutive Model}

The constitutive model used in this work is based on delevopments discussed elsewhere in the literature (Hartl and Lagoudas, 2009). It considers an infinitesimal strain formulation, where the total strain is additively decomposed into elastic, thermal, phase transformation, and plastic contributions per

$$
\varepsilon=\mathcal{S}(\xi): \boldsymbol{\sigma}+\boldsymbol{\alpha}\left(T-T_{0}\right)+\varepsilon^{t}+\varepsilon^{p},
$$

where $\mathcal{S}$ denotes the compliance tensor and $\boldsymbol{\alpha}$ the thermal expansion coefficients. The evolutions of two inelastic strains $\varepsilon^{t}$ and $\varepsilon^{p}$ are given by

$$
\dot{\varepsilon^{t}}=\dot{\xi} \Lambda^{t} ; \quad \dot{\varepsilon}^{p}=\dot{\bar{\epsilon}}^{p} \Lambda^{p}
$$

where

$$
\boldsymbol{\Lambda}^{t}=\left\{\begin{array}{ll}
H^{\text {cur }}\left(\bar{\sigma}_{t}^{e f f}\right) \frac{3}{2} \frac{\boldsymbol{\sigma}_{t}^{e f f^{\prime}}}{\bar{\sigma}_{t}^{e f f}} ; & \dot{\xi}>0 \\
\frac{\varepsilon^{t-r}}{\xi^{r}} & ; \quad \dot{\xi}<0
\end{array} ; \quad \boldsymbol{\Lambda}^{p}=\frac{3}{2} \frac{\boldsymbol{\sigma}_{p}^{e f f^{\prime}}}{\bar{\sigma}_{p}^{e f f}} .\right.
$$

The inelastic processes evolve in the direction of the deviatoric part of the two effective stresses $\left(\boldsymbol{\sigma}_{t}^{e f f}=\boldsymbol{\sigma}+\boldsymbol{\beta}\right.$ for the transformation strain and $\boldsymbol{\sigma}_{p}^{e f f}=\boldsymbol{\sigma}-\boldsymbol{\beta}$ for the plastic strain). The scalar term $\bar{\sigma}^{\text {eff }}$ denotes the Mises equivalent of the effective stress specified. The function $H^{\text {cur }}\left(\bar{\sigma}_{t}^{\text {eff }}\right)$ denotes the maximum uniaxial transformation strain generated during complete transformation from austenite to martensite under uniaxial loading at a constant stress level $\bar{\sigma}_{t}^{e f f}$. The form of the transformation strain evolution equation during reverse transformation ensures that all transformation strain is recovered.

\footnotetext{
${ }^{a}$ e-mail: lagoudas@aero.tamu.edu
} 
In the current formulation, the back stress is assumed to be an explicit function of $\varepsilon^{p}$ and $\xi$, given as

$$
\boldsymbol{\beta}=K_{\beta}(\xi) \varepsilon^{p}+\boldsymbol{\beta}_{0},
$$

where $K_{\beta}(\xi)$ is the kinematic hardening modulus (varying linearly during transformation), and $\boldsymbol{\beta}_{0}$ denotes an initial back stress in the reference configuration.

The criterion that determines when transformation begins is given as

$$
\begin{array}{lll}
\dot{\xi} \geq 0 ; & \Phi^{t}=\pi^{t}-Y^{t}(\sigma) \leq 0 ; & \dot{\xi} \Phi^{t}=0, \\
\dot{\xi} \leq 0 ; & \Phi^{t}=-\pi^{t}-Y^{t}(\sigma) \leq 0 ; & \dot{\xi} \Phi^{t}=0,
\end{array}
$$

where the thermodynamic driving force for transformation is given as ${ }^{1}$

$$
\pi^{t}=(\boldsymbol{\sigma}+\boldsymbol{\beta}): \boldsymbol{\Lambda}^{t}+\frac{1}{2} \boldsymbol{\sigma}: \widetilde{\mathcal{S}} \boldsymbol{\sigma}+\rho \tilde{s}_{0} T-\rho \tilde{u}_{0}-\frac{1}{2} \tilde{K}_{\beta} \varepsilon^{p}: \varepsilon^{p}-f^{t}(\xi) .
$$

For plastic yielding, the criterion is

$$
\dot{\bar{\epsilon}}^{p} \geq 0 ; \quad \Phi^{p}=\pi^{p}-Y^{p} \leq 0 ; \quad \dot{\bar{\epsilon}}^{p} \Phi^{p}=0 .
$$

where the thermodynamic driving force is given as

$$
\pi^{p}=(\boldsymbol{\sigma}-\boldsymbol{\beta}): \boldsymbol{\Lambda}^{p}-\left(f_{A}^{p}\left(\bar{\epsilon}^{p}\right)+\xi \tilde{f}^{p}\left(\bar{\epsilon}^{p}\right)\right)
$$

The transformation hardening function $f^{t}(\xi)$ is given by

$$
f^{t}(\xi)=\left\{\begin{array}{l}
\frac{1}{2} a_{1}\left(1+\xi^{n_{1}}-(1-\xi)^{n_{2}}\right)+a_{3} ; \dot{\xi}>0 \\
\frac{1}{2} a_{2}\left(1+\xi^{n_{3}}-(1-\xi)^{n_{4}}\right)-a_{3} ; \dot{\xi}<0
\end{array} .\right.
$$

This transformation hardening form provides a smooth transition from thermoelastic behavior to transformation, the degree of smoothness being based on the selection of the parameters $n_{i}$. The plastic hardening functions $f^{p}\left(\bar{\epsilon}^{p}\right)$ for austenite and martensite are given as

$$
f_{\gamma}^{p}\left(\bar{\epsilon}^{p}\right)=\left(Y_{m}^{\gamma}-Y_{0}^{\gamma}\right)\left(1-e^{-C_{H} \bar{\epsilon}^{p}}\right)-K_{\beta}^{\gamma} \bar{\epsilon}^{p}, \quad \gamma=A, M .
$$

\section{Model Calibration}

Considering these constitutive relations, the following model parameters must be calibrated to capture the phase transformation: $i$ ) the elastic compliance tensors for pure martensite and austenite (defined using Young's moduli $E^{M}$ and $E^{A}$ and Poisson's ratios $\nu^{M}$ and $\nu^{A}$ for isotropic phases), ii) the functional description of the maximum transformation strain $\left(H^{\text {cur }}(\sigma)\right)$, and iii) five additional model parameters $\left(\rho \tilde{s}_{0}, \rho \tilde{u}_{0}, a_{1}, a_{2}, a_{3}\right)$ and the form of $Y^{t}(\sigma)$. Note that the hardening coefficients $n_{1}-n_{4}$ are directly chosen to best fit the four corners of the transformation hysteresis. It can be shown that the five remaining model parameters and $Y^{t}(\sigma)$ can be calibrated from six experimental parameters often used to quantify the SMA phase diagram: four transformation temperatures and two slopes (the stress influence coefficients, $C^{A}$ and $C^{M}$ ).

In the case that the process of plastic yield is also considered, the yielding behavior of pure austenite and pure martensite must also be characterized (giving $Y_{0}, Y_{m}, K_{\beta}$, etc. for the two phases). Consideration of the plastic back stress in the reference configuration proved to be especially important in the accurate modeling of transformation anisotropy observed in this drawn wire.

\footnotetext{
1 Throughout this work we will adopt the notation that, for any quantity $x$ defined in the pure austenitic phase $\left(x^{A}\right)$ and pure martensitic phase $\left(x^{M}\right)$, the difference is given as $\tilde{x}=x^{M}-x^{A}$.
} 
The minimum number of experiments needed to fully calibrate the transformation relations in the current constitutive model is then two uniaxial isothermal pseudoelastic (or superelastic) tests, which provide elastic properties and an approximation of the phase diagram, and one isothermal combined tension-torsion experiment, which provides some information on the threedimensional texture of the material and defines $\boldsymbol{\beta}_{0}$. However, to increase calibration accuracy, the authors considered the simulation of many isothermal experiments (uniaxial and combined tension-torsion), and then iterated over the set of model parameters until the sum of the squares of the prediction errors across all considered tests was minimized. Errors in tension-torsion predictions were assigned double weight relative to errors in uniaxial predictions, as the prediction of this combined response was considered an especially unique and important contribution of the current RoundRobin.

\section{Simulation Results and Discussion}

Four classes of experimental results were provided to SMA modelers participating in the S3TRoundRobin effort. These included: $i$ ) isothermal tensile pseudoelastic hystereses obtained at various temperatures (both full and partial loops), $i i$ ) constant stress tensile actuation hystereses (full loops), iii) isothermal combined tension-torsion hystereses (non-proportional, full loops), and $i v$ ) tensile "stress recovery" tests for different applied strains.

For each of the experimental classes considered, analytical results were obtained from finite element analysis of a $1.0 \mathrm{~mm}$ long segment of the SMA wire, which was $0.1 \mathrm{~mm}$ in diameter. A total of 320 axisymmetric elements (with twist) were used in the model, where one end of the segment was fully kinematically constrained while the other was subjected to the forces required to simulate the various experiments. The temperature field was specified in a spatially

Table 1. Material properties needed for the new SMA-plastic yield model as derived from tensile experiments on equiatomic NiTi (see the work of Hartl and Lagoudas (2009) for a description of properties)

\begin{tabular}{|c|c|c|c|}
\hline Parameter & Value & Parameter & Value \\
\hline \multicolumn{4}{|c|}{ (Thermoelastic Properties) } \\
\hline$E^{A}$ & $50 \mathrm{GPA}$ & $\nu^{M}=\nu^{A}$ & $0.33^{*}$ \\
\hline$E^{M}$ & $26.5 \mathrm{GPA}$ & $\alpha^{M}=\alpha^{M}$ & $10 e-6 /{ }^{\circ} \mathrm{C}^{*}$ \\
\hline \multicolumn{4}{|c|}{ (Phase Diagram Properties) } \\
\hline$M_{s}$ & $-67{ }^{\circ} \mathrm{C}$ & $\left.C^{A}\right|_{\sigma=150 M P a}$ & $7.4 \mathrm{MPa} /{ }^{\circ} \mathrm{C}$ \\
\hline$M_{f}$ & $-92{ }^{\circ} \mathrm{C}$ & $\left.C^{M}\right|_{\sigma=150 M P a}$ & $6.0 \mathrm{MPa} /{ }^{\circ} \mathrm{C}$ \\
\hline$A_{s}$ & $-44^{\circ} \mathrm{C}$ & & \\
\hline$A_{f}$ & $-32{ }^{\circ} \mathrm{C}$ & & \\
\hline \multicolumn{4}{|c|}{ (Transformation Strain Properties) } \\
\hline$H^{\text {cur }}(\sigma)=H$ & $\approx 0.047$ & & \\
\hline \multicolumn{4}{|c|}{ (Smooth Hardening Properties) } \\
\hline$n_{1}$ & 1.0 & $n_{2}$ & 1.0 \\
\hline$n_{3}$ & 1.0 & $n_{4}$ & 1.0 \\
\hline \multicolumn{4}{|c|}{ (Plastic Modeling Constants) } \\
\hline$Y_{0}{ }^{A}$ & $750 \mathrm{MPa}^{* *}$ & $C_{H}$ & 80 \\
\hline$Y_{0}^{M}$ & $750 \mathrm{MPa}^{* *}$ & $K_{\beta}{ }^{A}$ & $11.3 \mathrm{GPa}$ \\
\hline$Y_{m}{ }^{A}$ & $1200 \mathrm{MPa}$ & $K_{\beta}{ }^{M}$ & $8.3 \mathrm{GPa}$ \\
\hline$Y_{m}^{M}$ & $1200 \mathrm{MPa}$ & $\bar{\epsilon}_{\text {crit }}^{p}$ & 0.022 \\
\hline$\left[\beta_{0}\right]_{11}$ & $150 \mathrm{MPa}$ & {$\left[\beta_{0}\right]_{22}$} & $-75 \mathrm{MPa}$ \\
\hline$\left[\beta_{0}\right]_{33}$ & $-75 \mathrm{MPa}$ & {$\left[\beta_{0}\right]_{12}=\left[\beta_{0}\right]_{23}=\left[\beta_{0}\right]_{31}$} & $0 \mathrm{MPa}$ \\
\hline
\end{tabular}

${ }^{*}$ Nominal values taken from literature $\quad{ }^{* *}$ Used only during stress recovery simulations, else $1000 \mathrm{MPa}$ 
homogeneous manner. For each loading path, model strengths and weaknesses were assessed based on comparisons of experimental response and analytical simulations/predictions ${ }^{2}$.

\subsection{Isothermal tensile pseudoelastic tests}

In Figure 1, simulations and experiments are presented for isothermal tensile tests performed at four different temperatures. Note that while experimental curves at seven constant temperatures were provided, only six are shown. Observations are as follows:

- The apparent elastic moduli of austenite and martensite that describe the linear response before and after transformation, respectively, are well captured. Further, the importance of considering the difference in these slopes is obvious. The tension-torsion results will also show that the shear modulus resulting from isotropic relations accurately predicts the torsional response.

- The overall strain width of the hysteresis is captured accurately.

- Finally, while a majority of the hysteresis area is captured for each of the six tests shown, it is clear that the calibrated model predicts greater hardening than is observed in the experimental results. It will be shown that the tension-torsion hardening, however, is accurately captured, and it is postulated that this discrepancy a consequence of transformation localization. Specifically, the nominally homogeneous stress distribution over the wire crosssection combined with the gripping methods results in stress localizations during uniaxial

2 It should be noted that the experimental data provided to the authors for calibration/simulation/comparison was not identical to that provide to other RoundRobin members at an earlier date. For example, it will be seen that the the tension-torsion loading paths do no lead to complete transformation in the current experimental data, while that provided to others corresponds to nearly complete transformation.
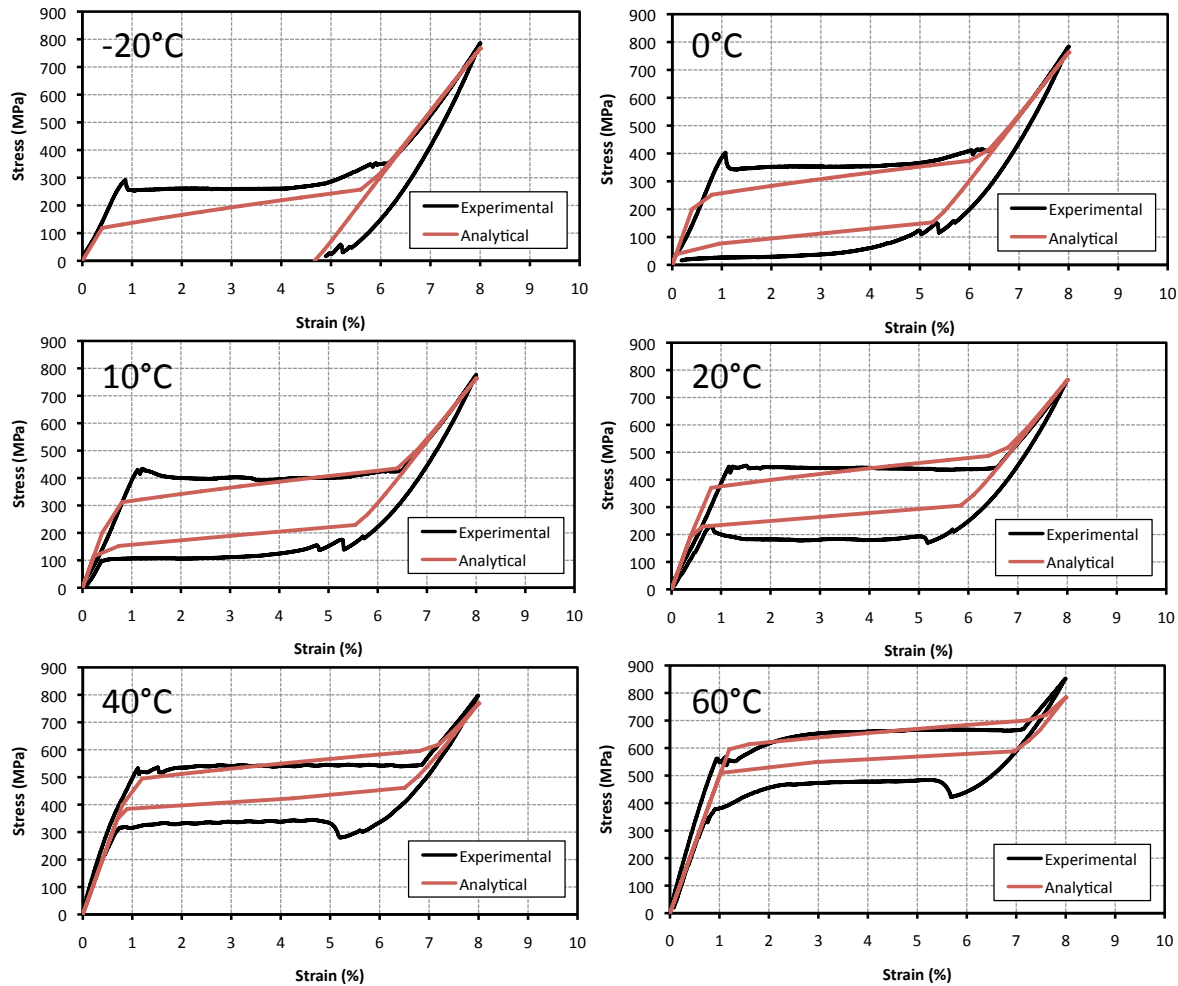

Fig. 1. Isothermal tensile pseudoelastic tests: experimental results and model predictions (test temperatures indicated). 
testing and associated reduction in transformation hardening. In the tension-torsion case, the stress gradient through the diameter of the wire prevents the initiation of transformation at a single point, instead distributing it along the entire length of the wire.

It should be noted that the partial loop experiments, though provided, were never simulated, and so the accuracy of the model in prediction this response feature is unknown.

\subsection{Constant stress tensile actuation tests}

Constant stress actuation data was provided for three different stress levels: 400, 450, and $500 \mathrm{MPa}$. The features of the constant stress tensile actuation tests (Figure 2) are each analogous to those previously described for the pseudoelastic cycles. In short, the elastic behavior is well captured (indicated by the accuracy of the strain predictions in pure austenite), and the hysteresis strain height is accurate. However, inaccuracies are again observed with regard to the transformation hardening, where the explanation based on localizations still applies due to the nominally homogenous stress state throughout the specimen cross-section.

\subsection{Isothermal combined tension-torsion tests}

This unique and important set of tests was based on the idea of non-proportional combinations of tensile and shear stress. Practically, such loading was imposed by first applying a constant axial load (by means of a hanging weight), and then imposing some twisting angle. This resulted in shear stresses and strains that varied with local radius. Such tests were performed at various ambient temperatures and considered various axial stresses. In every case, the maximum twist was $\approx 1.6 \mathrm{rad} / \mathrm{mm}$. The experimental results and analytical predictions for eight of the 68 provided cases (21 of which were simulated) are shown in Figure 3, where these have been selected to cover the range of imposed temperatures and axial stresses. Several key issue should be considered:

- In general, the predictions are quite accurate. This is due in large part to the method of calibration and the emphasis on matching these results in particular.
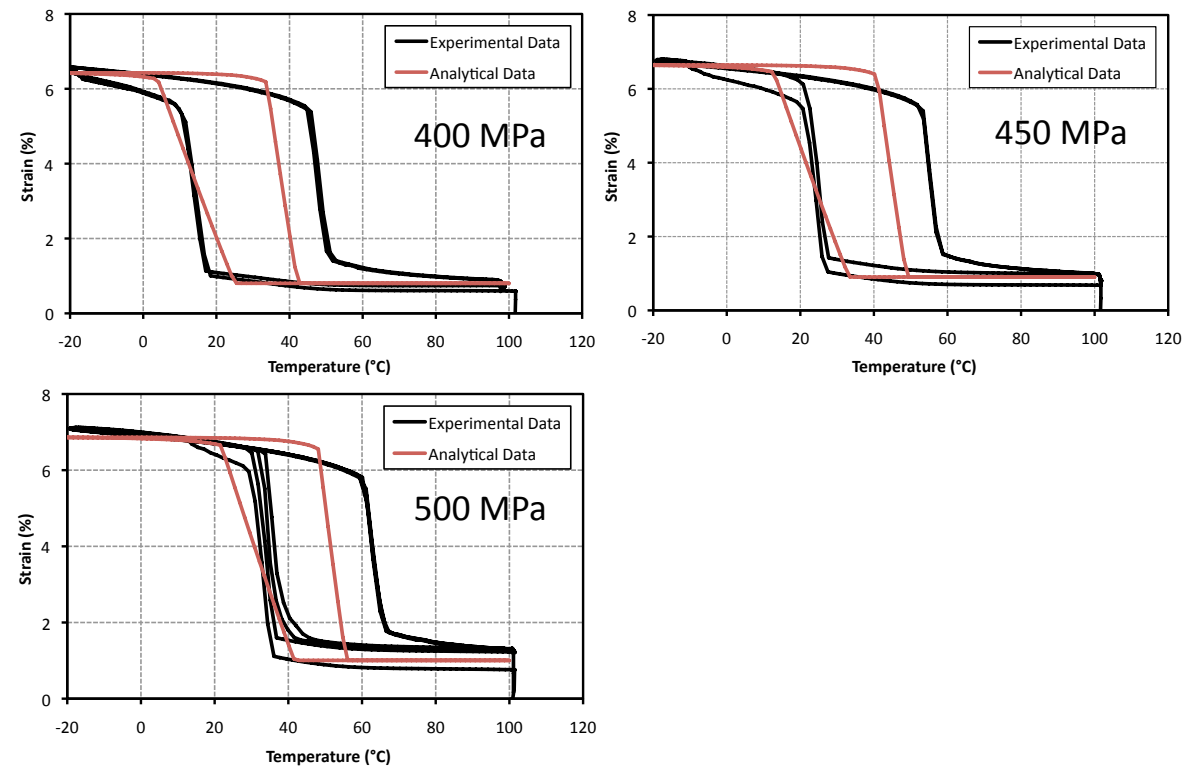

Fig. 2. Constant stress tensile tests: experimental results and model predictions (tensile test stresses indicated). 
- The ability to match both the previously discussed uniaxial results and the combined loading results with such accuracy using a model based on a $J_{2}$ (Mises-type) transformation criteria is only possible because of the unique contribution of the $\beta_{0}$ term, which accounts for the wire texture by providing internal biasing stresses.

\subsection{Tensile stress recovery tests}

The provided experimental database includes six recovery stress tests. These consist of isothermal uniaxial loading to three applied strains $(2,3.5$ and $5 \%$ ), either by stopping at the target strain during forward transformation (upper plateau), or during reverse transformation, after full forward transformation had been completed (lower plateau). In all six cases, this initial loading was applied at $20^{\circ} \mathrm{C}$. With the strain held constant at the target value, thermally-induced reverse transformation than led to large stresses as the material tried to recover oriented martensitic variants. Comparisons between experiments and model predictions are presented in Fig. 4, where both stress-strain and stress-temperature plots are provided for both $2 \%$ and both $5 \%$ cases. From these results we observe an overall agreement with the features of the response, although the same inaccuracies in capturing tensile pseudoelastic hardening remain. However,
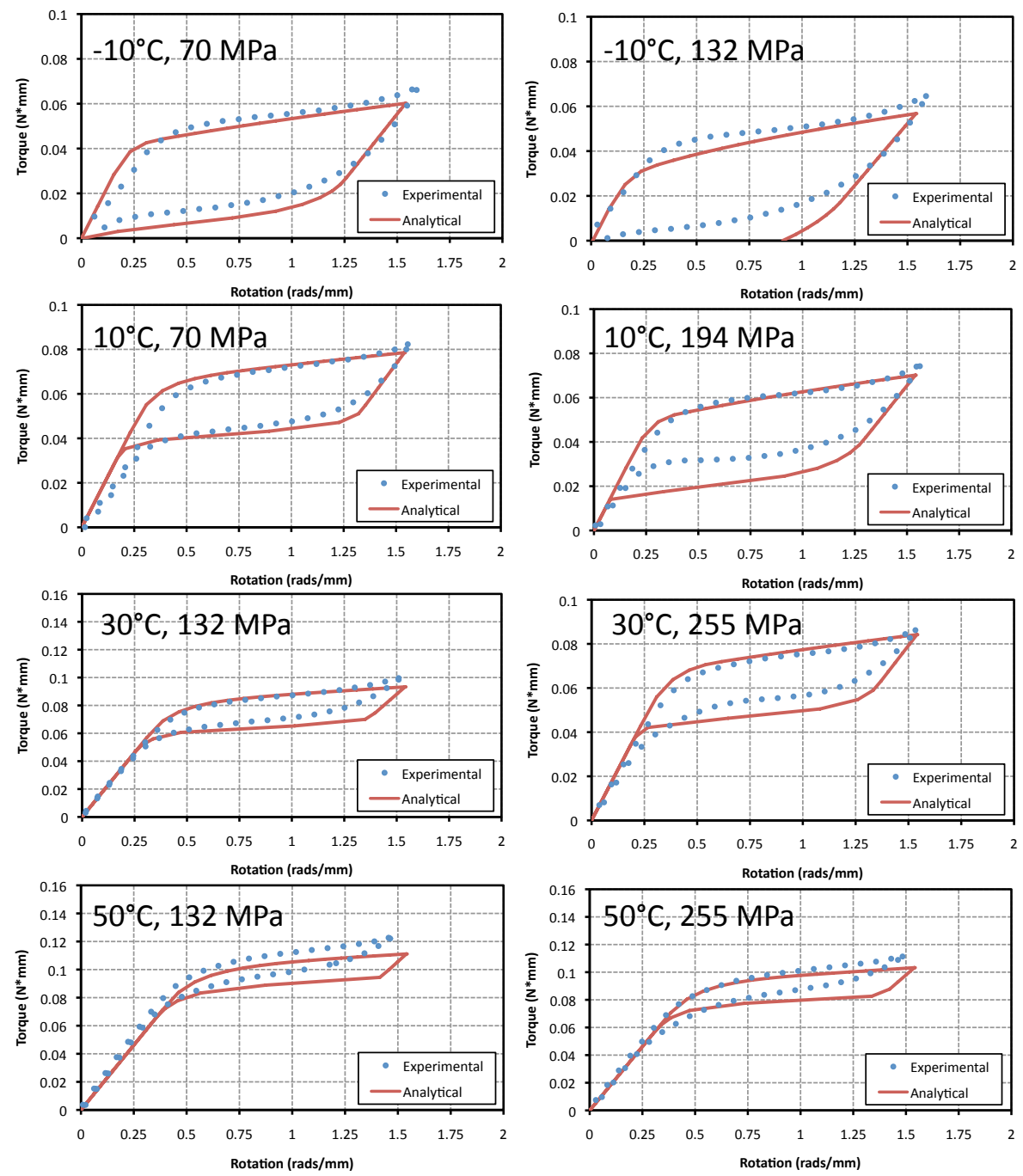

Fig. 3. Isothermal combined tension-torsion tests: experimental results and model predictions (test temperature and constant tensile stress indicated). 

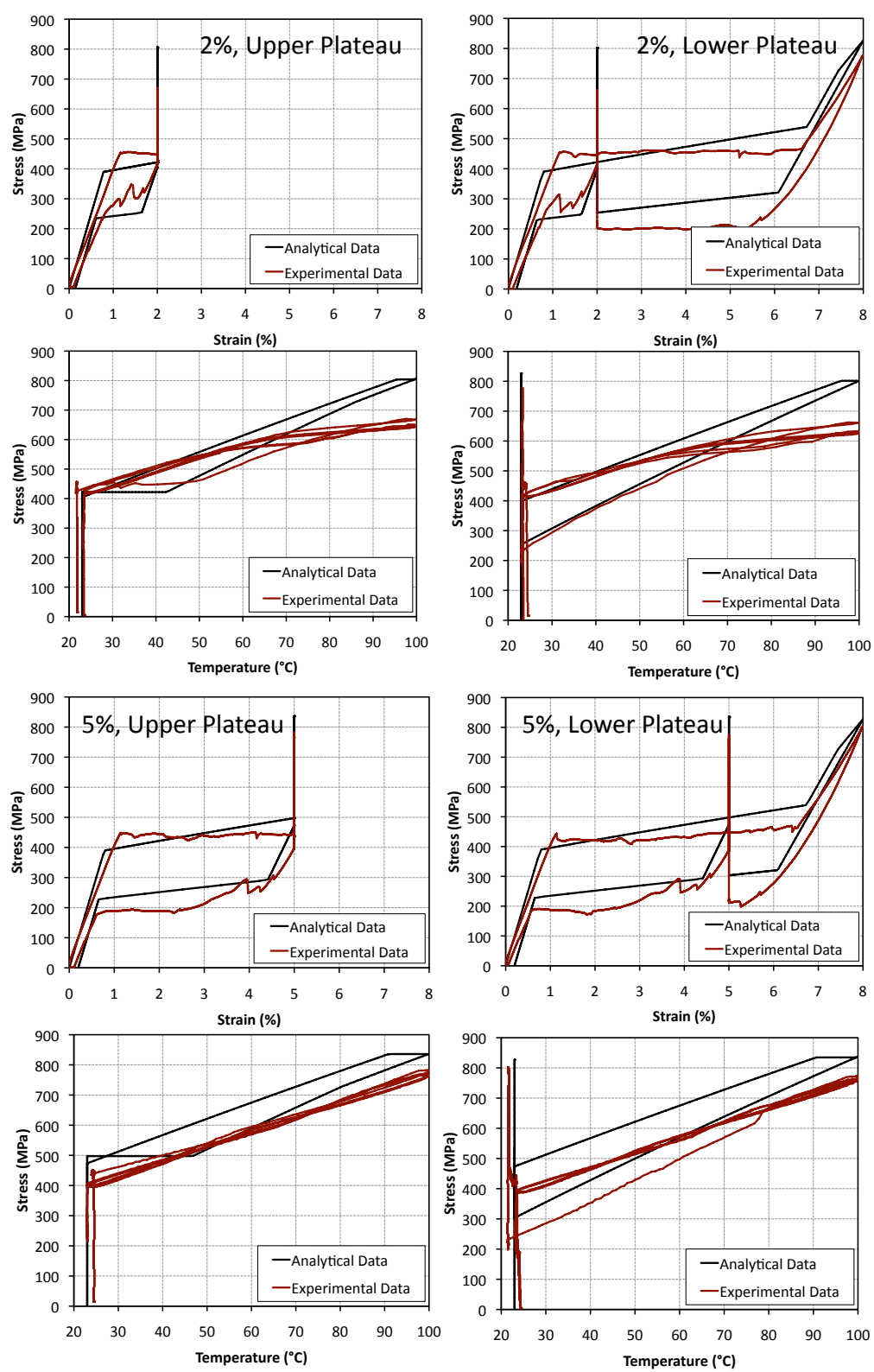

Fig. 4. Tensile stress recovery tests: experimental results and model predictions (fixed strain magnitude and plateau indicated).

it is clear that the stress-temperature behavior during the constrained recovery is well captured. Further, the unique capability of this model to capture plastic strain evolution allows prediction of the irrecoverable strain remaining at the completion of the thermomechanical cycle.

\section{References}

Hartl, D., Lagoudas, D., 2009. Constitutive modeling and structural analysis considering simultaneous phase transformation and plastic yield in shape memory alloys. Smart Materials and Structures 18 (1-17).

Hartl, D., Lagoudas, D., Mabe, J., Calkins, F., Mooney, J., 2009. Use of Ni60Ti shape memory alloy for active jet engine chevron application, Part II: Experimentally validated numerical analysis. Smart Materials and Structures 19 (1). 